for trend in women, respectively), and between the minor allele and overweight $(>25$ in $\mathrm{BMI}$, OR 1.52, 95\%Cl $1.072 .14, \mathrm{p}=0.02$ in men, OR $1.48,95 \% \mathrm{Cl} 1.16$ $1.95, p=0.01$ in women).

Logistic regression analysis showed a significant protective association in men with carriers of minor allele against low bone mass after an adjustment for age and $\mathrm{BMI}$ (OR $0.63,95 \% \mathrm{Cl} 0.440 .90, \mathrm{p}=0.01$ in men, not significant in women). Conclusion: Our study indicated significant associations of the polymorphism on FTO with BMI and bone mass among community dwelling men. The polymorphism may play a rule in a part of bone health with higher BMI and other beneficial functions.

References:

[1] N Engl J Med. 2015; 373: 895-907

Disclosure of Interests: None declared

DOI: 10.1136/annrheumdis-2020-eular.4050

\section{SAT0459 EVALUATION OF THE PREVALENCE AND THE MANAGEMENT OF OSTEOPOROTIC FRACTURES IN PATIENTS HOSPITALIZED AT NANCY UNIVERSITY HOSPITAL (FRANCE) IN 2017.}

A. Baccichetti ${ }^{1}$, P. L. Nguyen-Thi ${ }^{2}$, A. Blum ${ }^{3}$, D. Mainard ${ }^{4}$, F. Sirveaux ${ }^{4}$, L. Nace ${ }^{5}$,

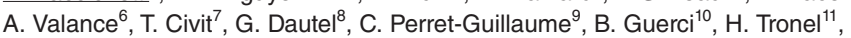
I. Chary Valckenaere ${ }^{1}$, D. Loeuille ${ }^{1}{ }^{1} \mathrm{CHU}$ Nancy, Rheumatology, Nancy, France; ${ }^{2} \mathrm{CHU}$ Nancy, Medical Evaluation, Nancy, France; ${ }^{3} \mathrm{CHU}$ Nancy, Radiology Guilloz, Nancy, France; ${ }^{4} \mathrm{CHU}$ Nancy, Orthopedic Surgery, Nancy, France $;{ }^{5} \mathrm{CHU}$ Nancy, Emergencies Unit, Nancy, France; ${ }^{6} \mathrm{CHU}$ Nancy, Post Emergencies Unit, Nancy, France; ${ }^{7} \mathrm{CHU}$ Nancy, Neurosurgery, Nancy, France; ${ }^{8} \mathrm{CHU}$ Nancy, Hand Surgery, Nancy, France; ${ }^{9} \mathrm{CHU}$ Nancy, Geriatrics, Nancy, France; ${ }^{10} \mathrm{CHU}$ Nancy, Endocrinology, Nancy, France; ${ }^{11} \mathrm{CHU}$ Nancy, Medical Information, Nancy, France

Background: Osteoporotic fractures are a major public health concern because of their consequences in morbidity, costs and mortality. In the meantime, historically postfracture osteoporosis medication use rates have been poor.

Objectives: The aim is to analyze the management of osteoporosis in patients hospitalized for osteoporotic fractures (OF) at Nancy University Hospital (France) in 2017

Methods: Total number of hospitalized patients and hospital stays were extracted by the Department of Medical Information (DIM) which selected departments with at least forty hospitalizations with Medical Unit Summary related to a diagnosis of fracture or osteoporosis. Hospitalizations not concerned by a recent OF were excluded. Data on fractures, patient characteristics, risk factors for OF and fall, management of osteoporosis, discharge status, stay duration, were studied from patient medical records. Prevalence of OF stays, management of osteoporosis and factors associated with duration of stay were analyzed.

Results: Out of a total of 153,840 hospitalizations, 918 hospitalizations (844 patients, mean age 74.5 years $\pm 13.6,74.5 \%$ women) concern an OF. The prevalence of hospitalizations for OF was $0.6 \%$ of total hospitalizations and $17.9 \%$ of total hospitalizations for fractures. Among the 844 patients, $85.7 \%$ had a severe fracture (vertebral fracture: $56.2 \%$, hip fracture: $24.1 \%$ ), $16.5 \%$ had a non-severe fracture, and $8.5 \%$ had a fracture cascade in the year. At discharge from hospital, $11.7 \%$ of patients received a specific treatment for osteoporosis. Longer stay duration was associated with age, severe fractures, Groll index and discharge status.

Conclusion: Nearly one hospitalized fracture in five is osteoporotic, while only one in ten patients is treated for osteoporosis. Stay duration increased with age and comorbidities. This encourages the development of early prevention, screening and treatment strategies for osteoporosis.

References:

[1] Hernlund E, Svedbom A, Ivergård M, Compston J, Cooper C, Stenmark J, et al. Osteoporosis in the European Union: medical management, epidemiology and economic burden. A report prepared in collaboration with the International Osteoporosis Foundation (IOF) and the European Federation of Pharmaceutical Industry Associations (EFPIA). Arch Osteoporos. 2013;8:136.

[2] Johnell O, Kanis JA. An estimate of the worldwide prevalence and disability associated with osteoporotic fractures. Osteoporos Int. 2006 Oct 19:17(12):1726-33.

[3] Giangregorio L, Papaioannou A, Cranney A, Zytaruk N, Adachi JD. Fragility Fractures and the Osteoporosis Care Gap: An International Phenomenon. Semin Arthritis Rheum. 2006 Apr;35(5):293-305.

Disclosure of Interests: None declared

DOI: 10.1136/annrheumdis-2020-eular.3366

\section{SAT0 460 INGESTION OF LEMON JUICE MAY MODULATE BONE METABOLISM.}

F. Bellone ${ }^{1}$, N. Morabito ${ }^{1}$, G. Pirisi ${ }^{1}$, S. Loddo ${ }^{1}$, R. Corsaro ${ }^{1}$, F. Corica ${ }^{1}$, A. Catalano ${ }^{1} .^{1}$ University of Messina, Department of Clinical and Experimental Medicine, Messina, Italy

Background: An association between bone health and consumption of citrus fruits have been previously reported; however, the effect of lemon juice on bone metabolism have not been explored yet.

Objectives: To investigate bone metabolic changes in postmenopausal women assuming lemon juice.

Methods: Participants were postmenopausal osteoporotic women without history of clinical fractures who agreed to enrich their diet with lemon juice (Act Lemon, Polenghi) over a 2-month period. The daily juice dose of $30 \mathrm{ml}$ we suggested was equivalent to one Sicilian organic lemon. Surrogate markers of bone formation as procollagen type $1 \mathrm{~N}$-propeptide (P1NP) and of bone resorption as C-terminal telopeptide of type I collagen (CTX), but also some regulators of bone metabolism as RANK-L, OPG, RANK-L/OPG ratio and sclerostin were assessed at baseline and then at 1 and 2 months after lemon juice administration. Controls were represented by a placebo group of age-matched osteoporotic postmenopausal women.

Results: 47 participants [mean age $60.2 \pm 4.1 \mathrm{yr}$.] completed the study, without reporting any adverse events. Lemon juice was well tolerated. Over the observation period modifications of bone metabolism occurred: we detected a decreased RANK-L/OPG ratio and increased CTX levels at all time points vs. baseline. Particularly, change at month-1 of sclerostin (versus baseline) has been positively associated with change at month- 1 and month- 2 of CTX ( $r=0.46$, $p=0.01$ and $r=0.43, p=0.01$, respectively). Change at month- 1 of OPG was positively associated with change at month-1 of P1NP $(r=0.49, p=0.006)$. Change at month-1 of RANKL/OPG has been related with variation at day 30 of P1NP $(r=-0.44, p=0.013)$. Variation of P1NP at month-1 was related with sclerostin variation at day $30(r=-0.56, p=0.02)$ and month- 2 vs. baseline value $(r=0.44$ $p=0.017)$ and with sclerostin variation between month- 1 and month- $2(r=0.69$, $\mathrm{p}<0.001$ ). Variation of P1NP between month-1 and month- 2 was associated with RANKL change at month-1 $(r=-0.35, p=0.05)$, with sclerostin change at month- 1 $(r=-0.49, p=0.008)$ and with sclerostin change between month-1 and month-2 $(r=0.41, p=0.028)$. At a multiple regression analysis the change of $P 1 N P$ between month-1 and month-2 was independently predicted by the change of sclerostin at month-1 ( $\beta=-1.5$, SE $0.5, p=0.006)$, after correcting for age, BMI and change of RANKL and CTX levels at month-1. No significant modifications raised from controls.

Conclusion: Drinking lemon juice may boost bone metabolic changes involving both bone resorption and bone formation.

Disclosure of Interests: None declared

DOI: 10.1136/annrheumdis-2020-eular.4579

\section{SAT0461 SHORT-TERM MONITORING OF DENOSUMAB EFFECT IN BREAST CANCER PATIENTS RECEIVING AROMATASE INHIBITORS USING REMS TECHNOLOGY ON LUMBAR SPINE}

E. Quarta ${ }^{1}$, D. Ciardo ${ }^{2}$, M. Ciccarese ${ }^{3}$, F. Conversano $^{2}$, M. DI Paola ${ }^{2}$, R. Forcignanò ${ }^{3}$, A. Grimaldi ${ }^{1}$, F. A. Lombardi ${ }^{2}$, M. Muratore ${ }^{1}$, P. Pisani ${ }^{2}$, S. Casciaro'. ' 'Galateo Hospital, ASL-LE, O.U. of Rheumatology, San Cesario di Lecce, Italy; ${ }^{2}$ National Research Council, Institute of Clinical Physiology, Lecce, Italy; ${ }^{3}$ Vito Fazzi Hospital, ASL-LE, O.U. of Oncology, Lecce, Italy

Background: Aromatase inhibitor (Al) therapy in women with estrogen receptor-positive $(\mathrm{ER}+)$ breast cancer $(\mathrm{BC})$ causes accelerated bone loss and increased risk of osteoporosis and fractures as side effects. Denosumab (i.e. $60 \mathrm{mg}$ twice a year) is a viable therapy against bone resorption, but the shortterm monitoring of bone mineral density (BMD) change with time is still an unmet clinical need, since the current techniques (including dual-energy $X$-ray absorptiometry, DXA) require 1-2 years between two consecutive measurements [1]. Radiofrequency Echographic Multi Spectrometry (REMS), with high performance in terms of precision and repeatability [2], might be used in this setting of patients for short-term monitoring of bone health-related parameters.

Objectives: The objective is the short-term monitoring of the effect of Als with/ without denosumab on bone health in BC patients using REMS and DXA scans at lumbar spine.

Methods: Post-menopausal ER+ BC patients treated with adjuvant Als were recruited. Two subgroups were identified, whether receiving also $60 \mathrm{mg}$ of denosumab therapy every 6 months or not (named Group A and Group B, respectively). All patients underwent baseline DXA and REMS lumbar spine scans at 
time T0, previous to the first Al therapy, and after 12 months (time T1). REMS scan only was repeated also at 18 months (T2), since a 6-month interval between two consecutive scans is not recommended for DXA. The bone mineral density (BMD) was measured with both techniques.

Results: Overall, 254 ER+ BC patients were enrolled (127 per group). The effect of denosumab on BMD is reported in Table. The BMD values obtained by DXA and REMS were not significantly different at T0 and T1, whereas the difference between Group A and B at T1 was statistically significant $(p<0.001)$ both for REMS and DXA. At T2, REMS confirmed the increasing trend of BMD for Group $A$ and the decreasing one for Group B, and the difference between groups was statistically significant $(p<0.001)$. For each time point and each group, there were not statistically significant differences between DXA and REMS.

Conclusion: Several studies have shown the effect of denosumab on BMD over a period not less than 2 years from the start of treatment. This study showed the feasibility of short-term follow-up using REMS lumbar spine scans at 6-month time steps.

References:

[1] Diez-Perez A et al, Aging Clin Exp Res 2019;31(10):1375-89

[2] Di Paola M et al, Osteoporos Int 2018;30:391-402

Table 1. BMD values, expressed as $\mathrm{g} / \mathrm{cm}^{2}$, measured by DXA and REMS for Group A (patients receiving Als only) and Group B (patients receiving Als and denosumab) at baseline (T0), 12 months (T1) and 18 months (T2) from the start of therapy. Results are presented as median values with $25^{\text {th }}$ and $75^{\text {th }}$ percentiles. P-values are obtained with a Mann-Whitney test.

\begin{tabular}{|c|c|c|c|c|c|c|}
\hline \multirow[b]{2}{*}{ Scan time } & \multicolumn{3}{|c|}{ DXA } & \multicolumn{3}{|c|}{ REMS } \\
\hline & Group A & Group B & $p$ & Group A & Group B & $p$ \\
\hline TO & $\begin{array}{c}0.840 \\
(0.719-0.959)\end{array}$ & $\begin{array}{c}0.867 \\
(0.723-0.958)\end{array}$ & 0.99 & $\begin{array}{c}0.833 \\
(0.708-0.949)\end{array}$ & $\begin{array}{c}0.855 \\
(0.714-0.973)\end{array}$ & 0.77 \\
\hline$T 1$ & $\begin{array}{c}0.823 \\
(0.702-0.944)\end{array}$ & $\begin{array}{c}0.889 \\
(0.749-0.990)\end{array}$ & 0.003 & $\begin{array}{c}0.819 \\
(0.691-0.927)\end{array}$ & $\begin{array}{c}0.887 \\
(0.740-1.018)\end{array}$ & $<0.001$ \\
\hline$T 2$ & - & - & - & $\begin{array}{c}0.801 \\
(0.679-0.909)\end{array}$ & $\begin{array}{c}0.899 \\
(0.754-1.020)\end{array}$ & $<0.001$ \\
\hline
\end{tabular}

Note: The authors D. Ciardo, M. Ciccarese, F. Conversano, M. Di Paola, R. Forcignanò, A. Grimaldi, F.A. Lombardi, M. Muratore and P. Pisani are listed in alphabetical order

Disclosure of Interests: None declared

DOI: 10.1136/annrheumdis-2020-eular.3806

\section{SAT0462 1 ASSESSMENT OF BONE MINERAL DENSITY IN INFLAMMATORY BOWEL DISEASE}

C. Daldoul ${ }^{1}$, N. El Amri ${ }^{1}$, K. Baccouche ${ }^{1}$, H. Zeglaoui ${ }^{1}$, E. Bouajina ${ }^{1} .{ }^{1}$ Farhat Hached Hospital, Rheumatology, Sousse, Tunisia

Background: Inflammatory bowel disease (IBD), including ulcerative colitis (UC) and Crohn's disease (CD), is considered as a risk factor of low bone mineral density (BMD). In fact, the prevalence of osteoporosis ranges from $17 \%$ to $41 \%$ in IBD patients. The possible contributing factors may include malabsorption, glucocorticoid treatment and coexisting comorbidities

Objectives: The purpose of our work was to determine the frequency and the determinants of osteoporosis in patients with IBD and to assess whether there is a difference in BMD status between UC and CD.

Methods: This is a retrospective study, over a period of 5 years (from January 2014 to December 2018) and including patients followed for IBD who had a measurement of BMD by DEXA. Clinical, anthropometric and densitometric data (BMD at the femoral and vertebral site) were recorded. The WHO criteria for the definition of osteoporosis and osteopenia were applied.

Results: One hundred and five patients were collected; among them 45 were men and 60 were women. The average age was 45.89 years old. The average body mass index (BMI) was $25.81 \mathrm{~kg} / \mathrm{m} 2$ [16.44-44.15]. CD and UC were diagnosed in respectively $57.1 \%$ and $42.9 \%$. A personal history of fragility fracture was noted in $4.8 \%$. Hypothyroidism was associated in one case. Early menopause was recorded in $7.6 \% .46 .8 \%$ patients were treated with corticosteroids. The mean BMD at the vertebral site was $1.023 \mathrm{~g} / \mathrm{cm} 3[0.569-1.489 \mathrm{~g} /$ $\mathrm{cm} 3$ ]. Mean BMD at the femoral site was $0.920 \mathrm{~g} / \mathrm{cm} 3[0.553-1.286 \mathrm{~g} / \mathrm{cm} 3$ ]. The mean T-score at the femoral site and the vertebral site were -1.04 SD and $-1.27 \mathrm{SD}$, respectively. Osteoporosis was found in $25.7 \%$ and osteopenia in $37.1 \%$. Osteoporosis among CD and UC patients was found in respectively $63 \%$ and $37 \%$. The age of the osteoporotic patients was significantly higher compared to those who were not osteoporotic ( 52.23 vs 43.67 years, $p=0.01$ ).
We found a significantly higher percentage of osteoporosis among men compared to women $(35.6 \%$ vs $18.3 \%, p=0.046)$. The $B M I$ was significantly lower in the osteoporotic patients (23.87 vs $26.48 \mathrm{~kg} / \mathrm{m} 2, \mathrm{p}=0.035)$ and we found a significant correlation between BMI and BMD at the femoral site $(p=0.01)$. No increase in the frequency of osteoporosis was noted in patients treated with corticosteroids $(27.9 \%$ vs $21.6 \%, p=0.479)$. Comparing the UC and CD patients, no difference was found in baseline characteristics, use of steroids or history of fracture. No statistically significant difference was found between UC and CD patients for osteoporosis $(p=0.478), B M D$ at the femoral site $(p=0.529)$ and at the vertebral site $(p=0.568)$.

Conclusion: Osteoporosis was found in $25.7 \%$ of IBD patients without any difference between CD and UC. This decline does not seem to be related to the treatment with corticosteroids but rather to the disease itself. Hence the interest of an early screening of this silent disease.

Disclosure of Interests: None declared

DOI: 10.1136/annrheumdis-2020-eular.6007

\section{SAT0463 SEVERE OSTEOPOROSIS IN COLOMBIAN PATIENTS WITH SYSTEMIC LUPUS ERYTHEMATOSUS}

J. C. Diaz-Coronado $^{1,2}$, S. Herrera ${ }^{1}$, D. Hernandez-Parra ${ }^{1}$, L. Betancur-

Vasquez $^{2}$, D. Gonzalez-Hurtado ${ }^{2}$, J. Gonzalez-Arango ${ }^{2}$, L. Uribe-Arango ${ }^{2}$, M. F. Saavedra Chacón ${ }^{2}$, J. Lacouture-Fierro ${ }^{2}$, S. Guerra-Zarama ${ }^{2}$, S. Monsalve ${ }^{2}$, J. D. Serna Giraldo ${ }^{2}$, J. D. Serna ${ }^{2}$, J. Barbosa ${ }^{2}$, R. Pineda Tamayo ${ }^{1} .{ }^{1}$ Artmedica, Grupo de Información Clínica, Medellín, Colombia; ${ }^{2}$ CES University,

Departamento Medicina Interna, Medellín, Colombia

Background: Osteoporosis predominantly affects post-menopausal women There is an important percentage of the population that have additional risk factors that decrease bone mineral density. Patients with Systemic Lupus Erythematosus (SLE) have an increased risk for osteoporosis due to corticosteroid use and chronic inflammation. This population could have a higher prevalence of osteoporosis when compared to post-menopausal women of equal or older age. There is a paucity of information regarding bone mineral density and SLE in Latin America.

Objectives: To describe the prevalence and incidence of osteoporosis and osteoporotic fractures in a Colombian population with Systemic Lupus Erythematosus Methods: We collected 464 clinical records of patients who met either the American College of Rheumatology 1997 or Systemic Lupus International Collaborating Clinics (SLICC) 2012 classification criteria for systemic lupus erythematosus between January 2015 and June 2019. The clinical and immunoserological characteristics, and damage accrual were monitored for one year. The diagnosis of osteoporosis was confirmed with densitometry by energy $x$-ray absorptiometry (DXA) and the presence of fragility fractures according to the rheumatologist's report in the clinical history. The description of proportions and incidence rate of osteoporosis and fragility fracture is performed.

Results: The mean age was 45 years, $96.5 \%$ were women and the mean disease duration was 12 years. Others clinical characteristics in table 1 . The prevalence of osteoporosis was $13.8 \%$ with an incidence of 1.1 fractures / 100 person-months in the general population with SLE. In postmenopausal women, over 50 years the prevalence of osteoporosis was $28.4 \%$ with an incidence of 0.8 fractures / 100 months person. In the densitometric characteristics, the mean bone mineral density was $0.772 \mathrm{gr} / \mathrm{cm} 2$, T-score spine -2.9 and T-score femoral -2.6. SLEDAI mean 1.5 (SD 2.92) and SLICC mean 1.

Table 1. clinical characteristics

\begin{tabular}{lcc}
\hline & $\mathbf{n}$ & $\%$ \\
\hline Active Smoking & 83 & 17.9 \\
Premature gonadal failure & 8 & 1.7 \\
Lupic Nephritis & 178 & 38.4 \\
Proteinuria >2.5grams/24hours & 34 & 7.3 \\
End Stage Renal Disease & 16 & 3.4 \\
Anti-dsDNA & 146 & 31.4 \\
Anti-Sm & 110 & 23.7 \\
Anti-Ro & 138 & 29.7 \\
Prednisone Cumulative Dose & $2.8 \mathrm{gr}$ & 12 \\
Antimalarial & 57 &
\end{tabular}

Conclusion: Low bone mineral density and severe osteoporosis are prevalent in our cohort with SLE. We have found a fracture rate of 1080 per 100.000 people, which is well over what has been reported in the general population (53-443 per 100.000 people in women). Osteoporotic fractures are part of damage accrual 